\title{
The impact of quality of life on treatment preferences in multiple sclerosis patients
}

\author{
This article was published in the following Dove Press journal: \\ Patient Preference and Adherence \\ 19 October 2017 \\ Number of times this article has been viewed
}

\section{Gitte Lee Mortensen' Peter V Rasmussen ${ }^{2}$}

'Medical Anthropology Department, AnthroConsult, ${ }^{2}$ Department of Neurology, University Hospital of Aarhus, Aarhus C, Denmark
Correspondence: Gitte Lee Mortensen Medical Anthropology Department, AnthroConsult, Fynsgade 24, 8000 Aarhus C, Denmark

Tel +4523964252

Email glm@anthroconsult.dk
Introduction: Multiple sclerosis (MS) is a demyelinating disorder with an unpredictable and often disabling course. MS symptoms are very heterogeneous and may lead to reduced physical, cognitive, and psychosocial functioning decreasing patients' quality of life (QoL). Today, various disease-modifying treatments (DMTs) may prevent disease progression. However, it is increasingly complex to select the right therapy for a given patient and patient preferences should be considered when making treatment decisions. This study aimed to explore the main factors affecting patients' preferences regarding MS treatment and health care.

Methods: Five qualitative focus group interviews were carried out with a total of 40 participants from across Denmark. A semistructured question guide included questions that were identified in a systematic literature study about QoL and treatment preferences in patients with MS. The participants were asked to describe their disease experiences, their health-related QoL, and reasons behind their preferences with regard to treatment and care. The data were analyzed using content analysis and a constructivist approach.

Results: The participants' physical, cognitive, and psychosocial QoL and functioning were reduced by disease symptoms, treatment side effects, and mode of administration. Their ability to uphold meaningful role functioning was crucial to their treatment priorities. The preeminence of anticipated efficacy, ie, the patients' hope that DMT might prevent disease deterioration in the future, was modified by their present QoL and functioning when ultimately framing their treatment preferences. There was an unmet information and support need from neurology clinics, particularly at the time of diagnosis.

Conclusion: The participants' treatment preferences were influenced by a matrix of treatment and QoL-related factors and evolved with time and along with personal and professional changes in life. The patients preferred to receive a clear recommendation of DMT from the neurologist taking into account their individual functioning and present QoL priorities.

Keywords: disease-modifying therapy, patient perspectives, quality of life, qualitative research, decision-making, needs

\section{Introduction}

Multiple sclerosis (MS) is a disorder of the central nervous system characterized by inflammation, demyelination, and degeneration. The majority of MS patients experience relapses and remissions of neurological symptoms. Independently of acute relapses, gradual disease deterioration (progression) tends to set in over time. ${ }^{1}$ Approximately $85 \%$ of MS patients have a relapsing-remitting course at onset (RRMS) of which the majority later turn into a secondary progressive MS (SPMS) characterized by progressive worsening with few or no relapses. The remaining $15 \%$ experience a primary progressive MS (PPMS) with steady progression from onset. MS is the most common nontraumatic, disabling neurological disorder in young 
adults with the first symptoms typically occurring between the age of 20 and 40 years. MS affects $>2.3$ million people worldwide and 13,000 in Denmark. Approximately twothirds are women. ${ }^{2,3}$

MS causes heterogeneous, fluctuating, and disabling symptoms, such as fatigue, mood changes, cognitive impairment, visual and sensory disturbances, pain, impaired mobility, spasticity, bladder and bowel dysfunction, sleep disorders, and sexual dysfunction. ${ }^{4-8}$ Symptoms may also combine to strengthen one another. ${ }^{9}$ The physical and psycho-social impacts on patients' health-related quality of life (HRQoL) and functioning vary accordingly. A number of studies have shown that disease progression, fatigue, pain, and depression, in particular, act to reduce patients' HRQoL as well as their professional and social role functioning. ${ }^{4-6,10}$

During the last 2 decades, several disease-modifying treatments (DMTs) have been approved and selecting the right therapy for a given patient can be complex. ${ }^{11}$ DMTs act to reduce the number and severity of relapses in RRMS with varying (30\%-70\%) efficacy. As the natural disease course in RRMS is unpredictable with respect to the number and severity of relapses and subsequent symptoms, the benefit of treatment for the individual patient is thus unknown. Besides a clinical evaluation of the benefits and risks associated with the different drugs, patient preferences regarding acceptable side effects, frequency, and mode of administration should be considered in treatment decisions. Previous studies have suggested that patients' treatment preferences are related to their self-assessed HRQoL. ${ }^{12,13}$ However, the connections between patients' disease and treatment experiences and their treatment preferences have not been explored in depth.
Therefore, our aim was to explore which specific treatments may be preferable from MS patient perspectives using qualitative research methods, and why.

\section{Methods}

This study was based on qualitative focus group interviews with 40 participants invited to participate via the Facebook page of the Danish MS patient organization. Interested patients from across Denmark were encouraged to make contact for further information and screening after which they could volunteer to participate anonymously. As no medical records were collected and the data collection only constituted the participants' subjective descriptions of their disease experiences and treatment preferences, the study did not require ethics committee approval in Denmark. The participants provided written informed consent for this study. They were divided into five focus groups with the aim of creating confidential settings for discussions among peers with comparable treatment experiences. Group A consisted of recently diagnosed MS patients (maximum 18 months). Group B brought together patients diagnosed $>2$ years ago who had switched to another first-line DMT. In group C, patients had switched to second or later lines of DMT. Group D consisted of patients with SPMS and PPMS (not receiving DMT), and group E included patients having opted out of DMT. Besides the varying treatment experiences, the aim was to include a diversity of MS patients with respect to age and gender (Table 1).

The focus groups were set in a conference room in Copenhagen, Denmark, and lasted 2 hours. They were moderated by GLM using a semistructured question guide that was informed by a systematic study of literature about

Table I Participants' experiences with disease modifying therapy

\begin{tabular}{|c|c|c|c|c|}
\hline DMT & $\begin{array}{l}\text { Mode and frequency of } \\
\text { administration }\end{array}$ & $\begin{array}{l}\text { Number of participants having } \\
\text { received DMT at any time }\end{array}$ & $\begin{array}{l}\text { Number of participants } \\
\text { currently receiving DMT }\end{array}$ & $\begin{array}{l}\text { Not currently } \\
\text { receiving DMT }\end{array}$ \\
\hline Alemtuzumab & Annual IV infusion course & $\mathrm{I}$ & I & \\
\hline Dimethyl fumarate & Two daily tablets & 9 & 4 & \\
\hline Fingolimod & One daily tablet & 11 & 5 & \\
\hline Glatiramer acetate & Daily SC injection & 11 & 2 & \\
\hline Interferon beta-la (Avonex) & Weekly IM injection & 21 & 0 & \\
\hline Interferon beta-Ib (Betaferon) & SC injection every second day & 6 & 0 & \\
\hline Interferon beta-Ib (Extavia) & SC injection every second day & I & 0 & \\
\hline Interferon beta-Ia (Rebif) & SC injection three times a week & 5 & I & \\
\hline Natalizumab & IV infusion every four weeks & 12 & 7 & \\
\hline Peginterferon beta-Ia & SC injection every second week & 2 & I & \\
\hline $\begin{array}{l}\text { Patients with PPMS }(n=3) \text { and } \\
\text { SPMS }(n=3)\end{array}$ & & (3 with SPMS previously in DMT) & 0 & 6 \\
\hline No DMT & & (7 previously in DMT) & 0 & 9 \\
\hline
\end{tabular}

Abbreviations: DMT, disease-modifying therapy; IM, intramuscular; IV, intravenous; PPMS, primary progressive multiple sclerosis; SC, subcutaneous; SPMS, secondary progressive multiple sclerosis. 
HRQoL and treatment preferences in MS patients. Following a funnel-shaped structure with open-ended questions, the interviews began with an inquiry into the participants' initial reactions to the MS diagnosis. They were then asked to describe their current symptoms after which the disease's physical, cognitive, psychosocial, and professional impacts on their quality of life (QoL) were discussed. Finally, the participants recounted their treatment experiences and explained their preferences with regard to DMT as well as additional care needs. ${ }^{14}$

The interviews were transcribed verbatim and analyzed using the NVivo 8 software (QSR International, Melbourne, QLD, Australia) and a combination of inductive content analysis and constructivist approach, ie, focusing on how the language used in the focus group discussions unveiled how patterns of meaning were created in social interaction among peers and in relation to each patients' personal context. ${ }^{15,16}$ This framework allowed us to investigate a diversity of statements in a manner that generated clusters of meaning around and about patient preferences in MS. It involved an examination of the terminology used to speak about this topic and how it is related to other issues. First, the transcripts were read through numerous times (independently, by three researchers) to get familiar with the data. Second, the data were coded (categorized) into moderator- and participantgenerated topics that were raised during the discussions. Some categories were collapsed; others were spilt up into separate categories. Third, the main themes within each topic were identified, and finally, recurrent connections between topics and themes were analyzed. Main topics and themes were discussed among GLM, the participating research assistant (IBM), and an independent qualitative researcher (SLM) until agreement was reached. This created a pattern of the participants' significant experiences with MS and how this related to their preferences for treatment and support.

\section{Results}

A total of 40 participants were involved in the study with 29 women and 11 men, aged between 18 and 63 years with almost half between 41 and 51 years (mean 44.4 years). They had been diagnosed between 2 months and 28 years ago, though half within the past 5 years (mean 7.8 years). Approximately half of the participants had children and/or a partner. Only five had full-time occupations, while 10-13 had flex jobs, were pensioners, or still had unresolved occupational status. Most of the latter were diagnosed within the past 5 years. The participants attended 12 different hospitals across Denmark. In total, 36 participants had received
11 different types of DMT, of whom 32 participants had tried two or more types (Table 1).

The following four main topics were discussed in the focus groups: the QoL impact of MS, treatment experiences, DMT treatment preferences, and nonmedical needs for care and support. Each topic is described below and exemplified with patient quotes.

\section{Patient perceptions of the QoL impact of MS}

In most of the participants, the MS diagnosis was followed by 2-3 years of distress and worries about the future. Fatigue and concerns about future relapses were depicted as especially burdensome, and most had experienced recurring emotional oversensitivity, depression, anxiety, and anger. The focus group discussions revealed that the diagnosis often involved a painful change of self-perception reducing the participants' psychosocial QoL: from being seen by oneself and others as healthy, normal, and able bodied to becoming sick, abnormal, and disabled. Some felt stigmatized as a consequence of using mobility aids or due to unstable gait - leading to potentially shameful misperceptions of intoxication.

\section{Patient quotes I. Psychosocial impact of MS}

I see a psychologist because of the diagnosis. The thing is, your identity gets so shaken. You go from being a super human being to "shit, I'm sick! Can I keep on being the same person?" The things that make up your identity are unsettled. To me, it's been good getting help to distinguish between what's me as a sick person and what's me as a person [27-year-old woman, group A].

I'd just met my wife [when he received a PPMS diagnosis] and back then, I was lively and cheerful and we went out dancing every night. All of a sudden, I couldn't do that anymore and I started worrying about what else I might soon not be able to do ... but the diagnosis led to us getting married. Up until then, we'd just been fooling around, but it suddenly became so serious ... I guess I worry more about it than she does. When you've gone from being what I was [having a physically demanding job] to what I am now ... in my eyes, I used to be a great and handsome guy who could do all sorts of things. There was nothing I couldn't do. Winter swim or ride my motorcycle, we just did it! It was kind of in my set-up that there's nothing you can't do. Then all of a sudden there was this other impression of my person. Right now, as long as I exercise, I can do certain things but what when I can't do that anymore? Where do we go from there? [56-year-old man, group D] 
You lose your professional identity. It's difficult to communicate with others when you don't have an occupational content to your life. When you go out, people ask you "so what are you doing?" So yes, you lose part of your identity until it is reconstructed and you a new social network. That's hard [63-year-old man, group E].

To me, being a relatively young man, it's difficult to introduce myself as a disabled pensioner. That's not something you do with a smile on your face [33-year-old man, group E].

In most cases, acceptance of the disease gradually settled in and with time MS became detached as "Something I have, not something I am" (woman, group A). As a man in group B explained: "After a while, you get better at not thinking about it all the time, while at the same time always taking it into account in your planning and prioritizing." With fatigue being a key symptom, the ability to plan and prioritize proved crucial to coping with living with MS.

The participants described how their QoL was impaired by common physical MS symptoms such as dizziness, numbness, bladder problems, pain, drop-foot, spasms, and heat sensitivity. Many also had cognitive and linguistic problems and a low threshold to stress. Altogether, the psychosocial, physical, and cognitive QoL aspects severely affected the participants' functioning in core life domains such as work, housekeeping, tending to children, and leisure activities. Consequently, MS affected the entire household: financially, practically, and emotionally, putting a strain on partner relations and children. Many participants felt that their social lives had diminished as they prioritized their partner, family, and occupation. The QoL impact of MS was thus not only related to the participants' individual well-being and functioning but to how this interfered with their total life situation including their age, family situation, social network, leisure activities, and occupation.

\section{Treatment experiences}

Treatment-related issues were a major factor influencing the participants' QoL and disease experiences. Most had positive experiences with symptom treatment, such as modafinil, fampridine, and baclofen, and negative experiences with side effects from DMT. Flu symptoms from interferon-beta and stomach problems in some teriflunomid recipients were described as particularly challenging. Some had needle phobia upsetting them several days before injections. The participants mentioned three main reasons for having switched DMT at some point, of which the most frequent was side effects or intolerance. Lack of efficacy was almost as common a reason for switching, and finally, approximately a third of patients had switched due to mode of administration (weariness of needles or difficulties finding a practicable injection site). All participants having opted entirely out of DMT said that this was mainly due to side effects. Despite subsequent relapses, they felt that their present QoL was more important than the risk of disease progression. Still, none rejected the idea that they might one day accept DMT (again).

Overall, participants with a shorter time since diagnosis were less actively involved in DMT decision making and preferred leaving the choice of DMT to the neurologist. Therefore, recently diagnosed patients had mainly switched on the neurologist's initiative due to efficacy issues. Over time, many became increasingly involved in treatment decisions and less accepting of side effects. Side effects then became an increasing reason for patients to want a switch as they wanted effective treatment, but not at significant expense of their daily QoL and functioning. Most of the participants not only wanted to be informed about treatment options but also to receive a clear treatment recommendation from the neurologist taking their personal situation and preferences into account. They felt unsafe and incompetent if given too much responsibility to choose the DMT themselves.

\section{Patient quotes 2. Patient experiences on the involvement in treatment decision making}

I am gradually learning that it's my responsibility alone. I told the neurologist that I think that's unfair and really difficult. I'm just me and you've got this long education behind you. You are giving me two choices [of DMT], but which is the best? Or should I stop taking any at all? He says 'I'll support you in whatever choice you make; you decide what you want". But how can I make that decision? Of course, I have some knowledge. I have searched for information and googled because I have had this for 20 years. But I'm no doctor. I sometimes wish I could get some more support instead of just "do you want the plague or the plague?" That's not a fair choice to the patient [45-year-old woman, group B].

My neurologist was really good at taking my circumstances in consideration. He considered my age, that I'm a woman and may want to have children in a few years' time. I was very happy about that [27-year-old woman, group A]

I didn't get any choices and I'm fine with that. I trust my neurologist. At first, he said "you will get Avonex [interferon beta-1a]" and then he explained about possible side effects to me. Later, because I didn't like the needle, he said "I think you're ready for Tecfidera [dimethyl fumarate]. 
I think that's just right for you". I didn't question that because I feel confident with him, he's run many tests on me and I believe he gives me what's best for me [45-yearold woman, group B].

Receiving clear advice about treatment was particularly crucial to patients with cognitive difficulties. Overall, most of the participants felt that they had a good dialogue with their neurologist, but this was slightly less the case with those having opted out of DMT, as well as some progressive patients who felt neglected. Although they did not receive DMT and associated check-ups, they still had medical issues, such as symptom management or needs for psycho-social support or mobility aids, that they felt required attention.

\section{The participants' treatment preferences}

In continuation of the above, the participants' described their preferences for DMT as related to efficacy, side effects, and mode of administration - all considering their previous treatment experiences, individual role functioning, and priorities such as child care and working ability - ie, a matrix of treatment and QoL-related factors that were weighed against each other. While efficacy was in principle decisive, they explained that this might ultimately be moderated by side effects or mode of administration. For instance, some had fear of needles leading to them reject any type of injectable DMT; others opted for the monthly natalizumab infusions due to its lack of daily administration and side effects, despite the risk of developing progressive multifocal leukoencephalopathy, a viral and often fatal brain disease. Some distrusted biomedicine in general or had such bad experiences with side effects and low efficacy that they rejected DMT all together. While temporary flushing or local skin reactions were mostly acceptable, severe flu symptoms, constant stomach problems, or needle phobia were considered too detrimental to their QoL and functioning.

\section{Patient quotes 3. Patient considerations related to their treatment preferences}

I weighed the risk of the side effects - the one there is with Tysabri [natalizumab] - and my quality of life. That's what it's all about for me. It's here and now that I'm alive, not in ten years, right? And that's why I have to say that it's now that my children need me and it is now that my husband must have me. It's now that we're together and we have to be able to do things. At least, if things go wrong at some point, which they don't, we have done our best. And yes, there's a risk, but there's a risk with all things in life, actually a higher risk with millions of other things than the medication I happen to take [39-year-old woman, group C].

To me, it's part of my regular routine to take a tablet in the evening. But I'm sure that if one day I was told that "look, you can inject yourself once a week and get a 90\% chance of not having any more attacks, or you can take a pill and it's 50\%". Well, then I'd just have to deal with the darn needle phobia. GLM: "So, efficacy is most important?" Well, yes, that is if you're not to have influenza symptoms. But of course, efficacy is very very important ...because, attacks stink, they really do, and you never know where and how they'll hit you next and how long they'll last [34-year-old woman, group C].

With regard to mode of administration, almost all participants preferred oral DMT to injections. Tablets were easy to take and recurrently described as less likely to making the person feel "pathologized" than injections. The negative feeling of "pathologization" might also be caused by severe side effects or hospital visits (natalizumab infusions). Frequency of administration affected the participants' preferences only in so far as they suffered from side effects or needle phobia.

\section{Patient quote 4. Pathologization due to injections and side effects of treatment}

I received Avonex shortly after the diagnosis and I took it for a bit more than a year. I was so bloody sick with sideeffects... I would inject myself on Monday evenings and then be in bed for one and a half to two days, and that was quite hard to explain to my two young children. I felt that I was deprived of two days every week and I didn't want that. GLM: "Did you tell the doctor?". Yes, and I said that I felt pathologized by those injections. I needed to prepare the things [the injections] and sometimes I bled all over the place and my children came running with tissues. I felt sicker, had pain everywhere and I was more tired. So, when we were going on a holiday, I deliberately chose to leave it all at home and I felt wonderful... and then when we got home, I decided to continue [not taking DMT] and I told the hospital. They said "well that's on your own account then", and I said "oh yes, and I feel damn good!" Later on, they offered me that new drug, Tecfidera, but I guess I got a bit scared because of the side effects I had earlier [39-year-old woman, group E].

\section{Additional care and support needs}

The final main topic pertained to the participants' nonmedical needs for care and support. To many (25), the patient organization had become the main source of information and support, 
though this was not the case at the time of diagnosis. The initial fear of disability, destabilized identity, and avoidance of other MS patients representing scary images of one's own potential future was described as delaying contact when the need was actually the greatest. The participants therefore expressed an unmet support need at the time of diagnosis, which they preferred be met by the neurology clinics, eg, by meetings informing them about symptom management, training, mobility aids, and socio-legal and psychological support options.

\section{Patient quote 5 . Information needs at diagnosis}

I think the information and counseling effort should come from the place where we get diagnosed. But not in a dismissive manner - like I get the impression that some of us have experienced. There has to be time to sit down, maybe not the same day, but soon thereafter. They could invite a group of newly diagnosed patients to a meeting informing them about the various options of support and advance information about what to expect in the near future, emotionally for instance, and some counseling about what to do with one's job, what to say or not to say, etc. All these things come tumbling when you get that message. Alright, "you don't die of it; you die with it". Fine, but the next thoughts concern work, life, marriage, the children, all those things. That's where it goes wrong for a lot of people... [56-year-old man, group D].
Also, cognitive impairment led some participants to request a personal health coordinator to help them navigate these options.

\section{Patient quote 6. Cognitive impairment leading to needs for health coordination}

Not only am I sick and my cognitive functioning is affected, but I also have to be the one to carry the information from A to B. Something is missing. Some kind of coordinator or just that $\mathrm{A}$ and $\mathrm{B}$ correspond directly, I don't mind. I just find it difficult to be the one to carry the information all the time. Because, certain things do slip my mind. On a good day, I may forget I can't walk. But on a bad day, I may forget that I'm actually disturbed in the head. And then when I have to tell things ... I could also use some help with applications and so forth to the public system, cause that's hard with the cognitive problems [47 year-old woman, group C].

\section{Discussion}

Qualitative explorations of MS patients' reflections on the trade-offs between mode and frequency of DMT administration, risks of side effects, and efficacy have been lacking. This study aimed to examine how MS patients' disease experiences and QoL might explain and relate to their treatment preferences and support needs. As illustrated in Figure 1,

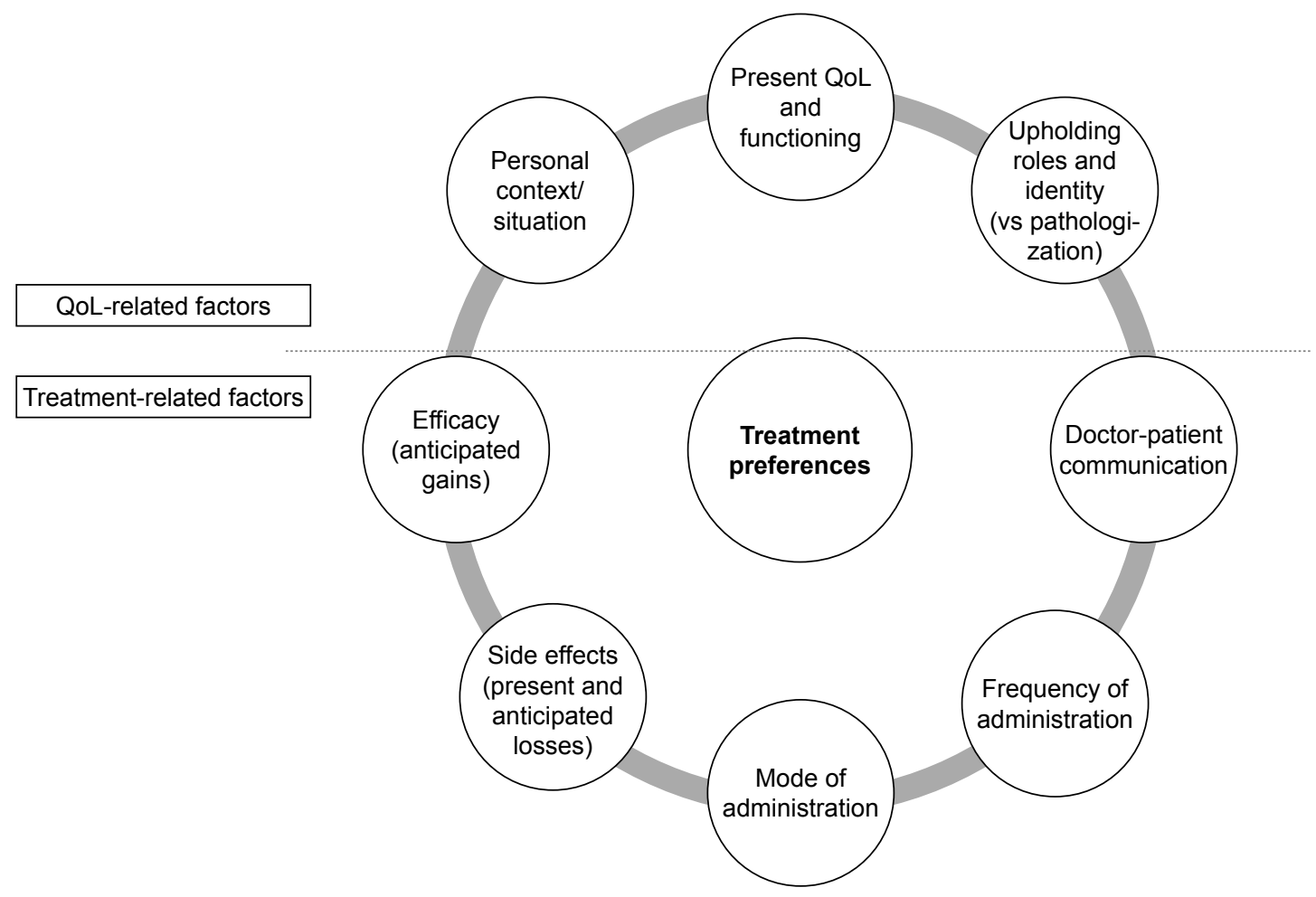

Figure I QoL and treatment-related factors influencing patients' treatment preferences. Abbreviation: QoL, quality of life. 
our participants' treatment preferences were affected by a matrix of treatment and QoL-related factors in which maintaining a positive self-image and meaningful roles was crucial. To them, expected efficacy was indeed important to their preference of DMT, but ultimately, their priorities were highly influenced by their present daily well-being and role functioning that might be compromised by a significant treatment burden. In line with other studies, they mostly preferred oral DMTs or, alternatively, monthly infusions. ${ }^{17,18}$ When not involving severe side effects, tablets were less likely to cause feeling pathologized, ie, enabling them to still feel like a parent, lover, or skilled professional, for instance, and less like a patient. The importance that the participants put on the daily disease burden is in line with studies suggesting that successful symptom management also enables MS patients to reconnect with former roles and activities, thereby preserving a crucial sense of their pre-MS self and identity. ${ }^{9,18,19}$

These MS patients' treatment preferences thus involved balancing their present and future QoL - anticipated gains and losses as described in the Health Belief Model (HBM). ${ }^{20}$ According to the HBM, health behavior is determined by personal perceptions about the perceived susceptibility and seriousness of the disease (including its impact on QoL) - together constituting the perceived threat - as well as the perceived benefits of and barriers to a given behavior. In order to take a certain kind of medicine, for instance, its value must be seen as greater than the losses. These four main constructs are modified by variables such as the cultural context, education, and past experiences, and later additions to the model include cues to action, motivation factors, and self-efficacy. ${ }^{21}$ It is a key feature of MS to involve uncertainty about future relapses and the efficacy of preventive medication. ${ }^{18}$ We believe that earlier findings that patients may be less focused on prevention of relapses (efficacy) than their clinicians ${ }^{9,19,22}$ may, in part, be explained by differing concepts of risk and severity. Patients may place comparatively greater importance on their present QoL and role functioning relative to the long-term gains. The importance of the individual context concurs with the finding that patients' DMT preferences shift with age and disease progression and that parenthood may impact on the acceptability of risks of side effects. ${ }^{18}$

As revealed by the frequent use of the concept of pathologization, identity issues may also act as barriers to support, particularly at the time of diagnosis. This created a need for hospital-based information and counseling that might also improve the care of patients who lack regular contact with the patient organization or hospital. Due to the inclusion method, our study may have over-represented high-functioning MS patients. It is likely, however, that these patients revealed needs that are even more pronounced in MS patients with lower QoL and functioning. The support gap at diagnosis may also particularly affect young patients and men whom we found difficult to include. Finally, while the use of qualitative methods allowed an in-depth understanding of MS patient perspectives on treatment, the low number of participants did not permit statistical analysis, eg, of the potential relations between treatment preferences and demographic factors. While the present study results are analytically generalizable, a quantitative follow-up survey may achieve statistical generalizability as well.

This study confirms that MS patients prefer shared decision making with regard to DMT and that later stage patients tend to be more actively involved. ${ }^{18,23}$ Our participants felt unsafe, however, if given too much of a free choice of DMT without a clear recommendation. Poor communication may also have contributed to some opting out of DMT. In addition to an evaluation of the benefits and risks associated with the different drugs, patient preferences regarding side effects, mode, and frequency of administration should be taken into account when making treatment decisions in MS throughout the course of the disease.

\section{Acknowledgments}

The authors wish to thank the Danish MS Society (Scleroseforeningen), particularly $\mathrm{PhD}$ and health policy consultant Lasse Skovgaard for his assistance with the inclusion of focus group participants and valuable methodological advice. GLM also thanks Biogen Idec, Denmark, for the unrestricted research grant funding the study. We are indebted to MA Ivan Bredbjerg Madsen for his research assistance at project preparations and data collection as well as anthropologist Steen Lee Mortensen for providing independent analysis and discussion of the qualitative data. Finally, we wish to thank Prof Emerit Michal Pugh and Philip Morley for copy editing the manuscript in English.

\section{Disclosure}

GLM is the owner of AnthroConsult who received an unrestricted research grant from Biogen Idec for the present study. AnthroConsult has previously received research grants and/or honoraria from Sanofi Pasteur MSD, Amgen, Pfizer, and Lundbeck A/S, though none related to studies of neurological disorders. PVR received a personal fee from Biogen Idec related to the present study. Within the past 2 years, he has participated in advisory board meetings with Biogen, Novartis, TEVA, Allergan, Roche, and Genzyme, acted as educator for TEVA, Allergan, and Novartis, and 
undertook letterbox assignments for Biogen. The authors report no other conflicts of interest in this work.

\section{References}

1. Lublin FD, Reingold SC. Defining the clinical course of multiple sclerosis: results of an international survey. National Multiple Sclerosis Society (USA) Advisory Committee on Clinical Trials of New Agents in Multiple Sclerosis. Neurology. 1996;46:907-911.

2. ms International Federation [homepage on the Internet]. Atlas of MS. 2013. Available from: www.msif.org. Accessed September 26, 2017.

3. Koch-Henriksen N, Magyari M, Laursen B. Registers of multiple sclerosis in Denmark. Acta Neurol Scand. 2015;132:4-10.

4. Coenen M, Basedow-Rajwich B, König N, Kesselring J, Cieza A. Functioning and disability in multiple sclerosis from the patient perspective. Chronic Illn. 2011;7(4):291-310.

5. Alschuler KN, Ehde DM, Jensen MP. Co-occurring depression and pain in multiple sclerosis. Phys Med Rehabil Clin N Am. 2013;24(4): 703-715.

6. Reese JP, Wienemann G, John A, et al. Preference-based health status in a German outpatient cohort with multiple sclerosis. Health Qual Life Outcomes. 2013;11:162

7. Flood S, Quinn H, Mendelowitz E, Marrie RA, Foley F. Predictors of fear of sexual rejection in individuals with multiple sclerosis. Mult Scler. 2014;20:388.

8. Stievano LP, Olival GS, Silva RA, et al. Validation survey of the impact of urinary incontinence (IIQ-7) and inventory of distress urogenital (UDI-6) - the short scales - in patients with multiple sclerosis. Arq Neuropsiquiatr. 2015;73(1):46-51.

9. Ziemssen T. Symptom management in patients with multiple sclerosis. J Neurol Sci. 2011;311(suppl 1):48-52.

10. Uccelli MM. The impact of multiple sclerosis on family members: a review of the literature. Neurodegener Dis Manag. 2014;4(2):177-185.

11. Soerensen PS. New management algorithms in multiple sclerosis. Curr Opin Neurol. 2014;27(3):246-259.
12. Grytten N, Aarseth JH, Espeseth K, et al. Health-related quality of life and disease-modifying treatment behaviour in relapsing-remitting multiple sclerosis - a multicentre cohort study. Acta Neurol Scand Suppl. 2012;195:51-57.

13. Solari A. Effective communication at the point of multiple sclerosis diagnosis. Mult Scler. 2014;20(4):397-402.

14. Halkier B. Fokusgrupper. Frederiksberg: Samfundslitteratur \& Roskilde Universitetsforlag; 2002.

15. Elo S, Kyngäs H. The qualitative content analysis. J Adv Nurs. 2008; $62: 107-115$

16. Winther Jørgensen M, Phillips L. Diskursanalyse som teori og metode. Roskilde: Roskilde University Press; 1999.

17. Utz KS, Hoog J, Wentrup A, et al. Patient preferences for diseasemodifying drugs in multiple sclerosis therapy: a choice-based conjoint analysis. Ther Adv Neurol Disord. 2014;7(6):263-275.

18. Wilson L, Loucks A, Bui C, et al. Patient centered decision making: use of conjoint analysis to determine risk-benefit trade-offs for preference sensitive treatment choices. J Neurol Sci. 2014;344(1-2):80-87.

19. Giovannoni G, Rhoades RW. Individualizing treatment goals and interventions for people with MS. Curr Opin Neurol. 2012;25(suppl): 20-27.

20. Rosenstock IM, Strecher VJ, Becker MH. Social learning theory and the health belief model. Health Educ Q. 1988;15(2):175-183.

21. Glanz K, Rimer BK, Viswanath K, editors. Health Behaviour and Health Education. Theory, Research, and Practice. 4th ed. San Francisco, CA: Jossey Bass; 2008.

22. Wicks P, Brandes D, Park J, Liakhovitski D, Koudinova T, Sasane R. Preferred features of oral treatments and predictors of non-adherence: two web-based choice experiments in multiple sclerosis patients. Interact J Med Res. 2015;4(1):e6.

23. Leone C, D'Amico E, Patti F. Do people with multiple sclerosis claim an 'active' role in the decision making process at start of first level therapy? Mult Scler. 2015;21:516-517.
Patient Preference and Adherence

\section{Publish your work in this journal}

Patient Preference and Adherence is an international, peer-reviewed, open access journal that focuses on the growing importance of patient preference and adherence throughout the therapeutic continuum. Patient satisfaction, acceptability, quality of life, compliance, persistence and their role in developing new therapeutic modalities and compounds to optimize
Dovepress

clinical outcomes for existing disease states are major areas of interest for the journal. This journal has been accepted for indexing on PubMed Central. The manuscript management system is completely online and includes a very quick and fair peer-review system, which is all easy to use. Visit http://www. dovepress.com/testimonials.php to read real quotes from published authors. 